\title{
THE GENERALIZED BERG THEOREM AND BDF-THEOREM
}

\author{
HUAXIN LIN
}

\begin{abstract}
Let $A$ be a separable simple $A F$-algebra with finitely many extreme traces. We give a necessary and sufficient condition for an essentially normal element $x \in M(A)$, i.e., $\pi(x)$ is normal $(\pi: M(A) \rightarrow M(A) / A$ is the quotient map), having the form $y+a$ for some normal element $y \in M(A)$ and $a \in A$. We also show that a normal element $x \in M(A)$ can be quasidiagonalized if and only if the Fredholm index $\operatorname{ind}(\lambda-x)=0$ for all $\lambda \notin$ $s p(\pi(x))$. In the case that $A$ is a simple $C^{*}$-algebra of real rank zero, with stable rank one and with continuous scale, $K_{1}(A)=0$, and $K_{0}(A)$ has countable rank, we show that a normal element $x \in M(A)$ with zero Fredholm index can be written as

$$
x=\sum_{n=1}^{\infty} \lambda_{n}\left(e_{n}-e_{n-1}\right)+a,
$$

where $\left\{e_{n}\right\}$ is an (increasing) approximate identity for $A$ consisting of projections, $\left\{\lambda_{n}\right\}$ is a bounded sequence of numbers and $a \in A$ with $\|a\|<\epsilon$ for any given $\epsilon>0$.
\end{abstract}

\section{INTRODUCTION}

Let $x \in B\left(l^{2}\right)$ be a normal operator. The Weyl-von Neumann-Berg theorem says that

$$
x=\sum_{n=1}^{\infty} \lambda_{n}\left(e_{n}-e_{n-1}\right)+a,
$$

where $\left\{e_{n}\right\}$ is an (increasing) approximate identity for $\mathcal{K}$ consisting of projections, $\left\{\lambda_{n}\right\}$ is a bounded sequence, $a \in \mathcal{K}$ and $\|a\|$ is smaller than any given positive number, where $\mathcal{K}$ is the $C^{*}$-algebra of compact operators on $l^{2}$. Note that $B\left(l^{2}\right)=M(\mathcal{K})$, the multiplier algebra of $\mathcal{K}$. Recently, Terry Loring and Jack Spielberg ([LS]) show that there are normal elements in $M(A)$ which are not quasidiagonalizable for some separable simple $A F$-algebra $A$ with a unique trace (up to scalar multiplication). Their counterexamples are based on the fact that a normal element in $M(A)$ may have nonzero Fredholm index. We will consider those separable simple $A F$-algebras with finite many (extreme) traces. We found that the Fredholm index is the only obstruction for quasidiagonality. In the case that $A$ has continuous scale and $K_{0}(A)$ has countable rank, we show that a normal element

Received by the editors July 27, 1993.

1991 Mathematics Subject Classification. Primary 46L05.

Key words and phrases. The Berg theorem, the BDF-theorem, weak (FN), $C^{*}$-algebra with real rank zero. 
$x \in M(A)$ can be written as

$$
x=\sum_{n=1}^{\infty} \lambda_{n}\left(e_{n}-e_{n-1}\right)+a,
$$

where $\left\{e_{n}\right\}$ is an approximate identity for $A$ consisting of projections, $\left\{\lambda_{n}\right\}$ is a bounded sequence of numbers and $a \in A$ with $\|a\|<\epsilon$ for any given $\epsilon>0$, if and only if the Fredholm index $i n d(\lambda-x)=0$ for all $\lambda \notin s p(\pi(x)(\pi: M(A) \rightarrow$ $M(A) / A$ is the quotient map). We also found that this obstruction is small, in fact, it is "infinitesimal", in the sense that ind $(\lambda-x)$ must be an infinitesimal element in $K_{0}(A)$, if $x$ is a normal element in $M(A)$. Therefore if $A$ is a separable simple $A F$-algebra with finite extreme traces and with no infinitesimal elements in $K_{0}(A)$, then every normal element in $M(A)$ can be quasidiagonalized. A related problem is when an essentially normal element (i.e. $\pi(x)$ is normal in $M(A) / A$, where $\pi: M(A) \rightarrow M(A) / A$ is the quotient map) $x \in M(A)$ can be written as $x=y+a$, where $y \in M(A)$ is a normal element and $a \in A$. One might imagine that $\operatorname{ind}(\lambda-x)=0$ for all $\lambda \notin s p(\pi(x))$ would be necessary and sufficient for $x=y+a$. However, we found that $\operatorname{ind}(\lambda-x)=0$ is neither necessary nor sufficient. It turns out, with some surprise, that an essentially normal element $x \in M(A)$ can be written as $x=y+a$ if and only if $\operatorname{ind}(\lambda-x)$ is an infinitesimal element in $K_{0}(A)$ for every $\lambda \notin s p(\pi(x))$ and the remaining $\Gamma(x)$ is zero, where $\Gamma$ is an index which will be defined in section 2 . This implies that every essentially normal element in $M(A)$ has the form $y+a$, where $y \in M(A)$ is normal and $a \in A$, if $A$ is a finite matroid algebra. One may view these results as some version of a generalized BDF-theorem for essentially normal elements.

\section{ACKNOWLEDGEMENTS}

This work was done while the author was visiting the State University of New York at Buffalo in 1992. The author would like to thank Lewis Coburn and Catherine Olsen for their efforts to make this visit possible. He is grateful to Terry Loring for sending him the preprint [LS].

\section{2. $C^{*}$-ALGEBRAS WITH WEAK $(\mathrm{FN})$}

Recall that a $C^{*}$-algebra $A$ is said to have real rank zero if the set of selfadjoint elements with finite spectrum is dense in $A_{s . a .}$. This class of $C^{*}$-algebras is currently under intensive study (see [BP], [BBEK], [BDR], [BKR], [Ell2]-[Ell3], [EE], $[\mathrm{G}],[\mathrm{R}]$, [Zh1]-[Zh6], [EGLP] and [Ln3]-[Ln13], etc.). In this section, we consider the problem when a normal element in a $C^{*}$-algebra of real rank zero can be approximated by normal elements in the algebra with finite spectrum. It is clear that the unitaries which are not connected with the identity in the unitary group can not be approximated by normal elments with finite spectra. The notion of weak (FU) (a $C^{*}$-algebra $A$ is said to have weak (FU) if the set of unitaries with finite spectra is dense in the connected component of a unitary group of $\tilde{A}$ containing the identity) was introduced by N. C. Phillips ([Ph]). It has been shown ([Ln7]) that every $C^{*}$-algebra with real rank zero has weak (FU). The problem for general normal elements is much more complicated. We begin with the following definition. 
Definition 2.1. Let $\phi: C(X) \rightarrow A$ be a monomorphism. The map $\phi$ induces a homomorphism

$$
\phi_{*}: K_{1}(C(X)) \rightarrow K_{1}(A) .
$$

In particular, if $x$ is a normal element in $A$, then $x$ gives a monomorphism $\phi$ : $C(s p(x)) \rightarrow A$. We define $\gamma(x)=\phi_{*}$. Let $I$ be a (closed and two sided) ideal of $A$. Suppose that $J$ is the kernel of the composition $\pi_{I} \circ \phi$. Then

$$
C(X) / J \cong C\left(X_{I}\right)
$$

for some compact subset $X_{I}$ of $X$. This induces a monomorphism

$$
\phi^{I}: C\left(X_{I}\right) \rightarrow A / I \text {. }
$$

We define

$$
\Gamma(\phi)=\left\{\left(\phi^{I}\right)_{*}: I \text { is an ideal of } A\right\}
$$

and $\Gamma(x)=\Gamma(\phi), \gamma^{I}(x)=\phi_{*}^{I}$, where $\phi: C(s p(x)) \rightarrow A$ is the map induced by the normal element $x \in A$. Notice $I$ could be $\{0\}$.

Definition 2.2 (cf. [Ln11]). A $C^{*}$-algebra $A$ is said to have weak $(F N)$ if every normal element $x \in A$ with $\Gamma(x)=0$ can be approximated by normal elements in $A$ with finite spectra. It has been shown in [Ln11] that all simple $A F$-algebras with countably many extreme traces, purely infinite simple $C^{*}$-algebras and separable simple $C^{*}$-algebras with real rank zero, stable rank one and weakly unperforated $K_{0}(A)$ of countable rank have weak $(\mathrm{FN})$. These algebras include UHF-algebras, matroid algebras, $C^{*}$-algebras of $O_{n}$, the Bunce-Deddens algebras and the irrational rotation algebras. In the case of $A F$-algebras and $O_{n}$, since the $K_{1}$-groups are trivial, these algebras in fact have (FN). In this section, we will show that some non-simple $C^{*}$-algebras have also weak (FN).

Added in proof (April 1996): It has been shown by the author in 1994 that every $A F$-algebra has (FN) and in 1996 by Friis and Rørdam that every $C^{*}$-algebra of real rank zero with connected unitary group and with property (IR) has (FN), and by the author that every simple $C^{*}$-algebra of real rank zero and with property (IR) has weak (FN). These include all simple $C^{*}$-algebras of real rank zero, stable rank one as well as all purely infinite simple $C^{*}$-algebras.

Remark 2.3. We are grateful to Terry Loring who pointed out that our original definition is not appropriate for non-simple $C^{*}$-algebra. Let $A=B \oplus C$, where $B$ is any (unital) $C^{*}$-algebra with real rank zero but nontrivial $K_{1}$-group and $C$ is any (unital) $C^{*}$-algebra with real rank zero. Let $u$ be a unitary in $B$ which is not connected to the identity in the unitary group of $B$. Set $x=u \oplus y$, where $y \in C$ is a normal element with spectrum $s p(y)=\bar{D}$ ( $\bar{D}$ is the closed unit disk). Clearly $\gamma(x)=0$, but $x$ can not be approximated by normal elements in $A$ with finite spectra. So maps $\left(\phi^{I}\right)_{*}$ are needed. It should also be noted that when $A$ is simple, $\Gamma=\gamma$.

Proposition 2.4. Let $A$ be a $C^{*}$-algebra with weak (FN). Then $\tilde{A}$ has weak (FN).

Proof. Let $x \in \tilde{A}$ be a normal element with $\Gamma(x)=0$. Then we may write $x=\lambda+a$ for some $a \in A$ and $\lambda \in \mathbf{C}$. Clearly, $a$ is a normal element with $\Gamma(a)=0$. The conclusion follows easily.

The following two lemmas are contained in $[\operatorname{Ln} 9,4]$, and $[\operatorname{Ln} 11,2.7]$. 
Lemma 2.5. Let $A$ be a $C^{*}$-algebra of real rank zero and $x \in A$ be a normal element with $\gamma(x)=0$. For any $\epsilon>0$, there is a $\delta>0$ such that if $p \in A$ is a projection and

$$
\|p x-x p\|<\delta / 2, \text { and }\|p x p-y\|<\delta / 2,
$$

where $y \in p A p$, and $\lambda(1-p)-(1-p) x(1-p) \in \operatorname{Inv}_{0}((1-p) A(1-p))$ if $\operatorname{dist}(\lambda, s p(x))$ $\geq \delta$, then $\lambda p-y \in \operatorname{Inv}_{0}(p A p)$, if $\operatorname{dist}(\lambda, s p(x)) \geq \delta$.

Proof. Suppose that $\operatorname{dist}(\lambda, s p(x)) \geq \delta$. We notice that

$$
\|(\lambda-[(1-p) x(1-p)+p x p])-(\lambda-x)\|<\delta .
$$

Therefore, by [Ln11, 2.3 and 2.7], $\lambda p-p x p \in \operatorname{Inv}_{0}(p A p)$. Now

$$
\|(\lambda p-y)-(\lambda p-p x p)\|<\delta / 2 .
$$

Then, by $[\operatorname{Ln} 9,4]$ and $[\operatorname{Ln} 11,2.7], \lambda p-y \in \operatorname{Inv}_{0}(p A p)$.

The following is proved in [Ln11, 3.12].

Theorem 2.6 $([\operatorname{Ln} 11,3.12])$. Let $\Omega$ be a compact subset of the plane. For any $\epsilon>0$, there exist $\delta>0$ and an integer $L$ such that for any (unital) $C^{*}$-algebra $A$ of real rank zero and a normal element $x$ in a $C^{*}$-algebra $B \supset A$ with $s p(x) \subset \Omega$, if $p \in A$ is a projection and if $p f(x), f(x) p \in A$ for any $f \in C(s p(x))$,

$$
\|p x-x p\|<\delta \text { and } \lambda p-p x p \in \operatorname{Inv}_{0}(p A p)
$$

for $\lambda \notin \Omega$, then there are normal elements $y \in M_{L}(p A p)$ and $z \in M_{L+1}(p A p)$ with finite spectrum $s p(y), s p(z) \subset \Omega$ such that

$$
\|p x p \oplus y-z\|<\epsilon .
$$

Lemma 2.7. Let $A$ be a $C^{*}$-algebra of real rank zero and $I$ be an ideal of $A$. Suppose that $A / I$ has weak (FN) and $I$ is a purely infinite simple $C^{*}$-algebra. Then $A$ has weak $(F N)$.

Proof. Let $x \in A$ be a normal element with $\Gamma(x)=0$. For any $\eta>0$, by $[\operatorname{Ln11}, 4.3]$ as in the first few lines in the proof of [Ln11, 4.4], there are $\lambda_{i} \in s p(x)=X$ and nonzero mutually orthogonal projections $p_{i}^{\prime} \in A$ such that

$$
\left\|x-\sum_{i=1}^{n} \lambda_{i} p_{i}^{\prime}-p^{\prime} x p^{\prime}\right\|<\eta / 4 \text { and }\left\|p^{\prime} x-x p^{\prime}\right\|<\eta / 4
$$

where $p^{\prime}=\left(1-\sum_{i=1}^{n} p_{i}^{\prime}\right)$ and $\left\{\lambda_{i}\right\}$ is $\eta / 16$-dense in $X$. Set

$$
I^{\perp}=\{a: a i=i a=0, \text { for } i \in I\} .
$$

From the proof of $[\operatorname{Ln} 11,4.3]$, if $p_{i}^{\prime} \notin I^{\perp}$, by taking a subprojection, one may assume that $p_{i}^{\prime} \in I$. Therefore, we may assume that $p_{i}=p_{i}^{\prime} \in I, i=1,2, \ldots, k$, and $\left\{\lambda_{i}\right\}_{i=1}^{k}$ is $\eta / 16$-dense in $s p(c x)$, where $c$ is the central open projection in $A^{* *}$ corresponding to the ideal $I$. Set $p=1-\sum_{i=1}^{k} p_{i}$. From the proof of $[\operatorname{Ln} 11,3.4]$ we have

$$
\left\|x-\sum_{i=1}^{k} \lambda_{i} p_{i}-p x p\right\|<\eta / 4 \text { and }\|p x-x p\|<\eta / 4 .
$$


Set $y=p x p$. Let $\pi: A \rightarrow A / I$ be the quotient map. Then $\pi(y)=\pi(x)$. By our assumption, we may assume that

$$
\left\|\pi(y)-\sum_{j=1}^{n} \alpha_{j} \bar{q}_{j}\right\|<\eta / 8,
$$

where $\bar{q}_{j}$ are mutually orthogonal projections in $p A p / I \cong p A p / p I p$ and $\alpha_{j} \in$ $s p(\pi(x))$. Since $p A p$ has real rank zero, by [Zh2, 2.3], every projection in $p A p / p I p$ lifts to a projection in $p A p$. Therefore there is $a \in p I p$ such that

$$
\left\|y-\sum_{j=1}^{n} \alpha_{j} q_{j}-a\right\|<\eta / 8
$$

where $q_{j}$ are mutually orthogonal projections in $p A p$. Since $I$ has real rank zero, $I$ has an approximate identity consisting of projections. Therefore there are projections $e_{j} \in q_{j} I q_{j}$ such that

$$
\|e a e-a\|<\eta / 16, \quad\|e y-y e\|<3 \eta / 16
$$

and

$$
\| \text { eye }-\sum_{j=1}^{n} \alpha_{j} e_{j}-e a e \|<\eta / 4,
$$

where $e=\sum_{j=1}^{n} e_{j}$. This also implies that

$$
\|e x-x e\|<\eta / 2 .
$$

Set $z=\sum_{j=1}^{n} \alpha_{j} e_{j}-e a e$. Then

$$
\|z-e x e\|<\eta / 2 .
$$

Notice that $c x$ is a normal element (in $A^{* *}$ ) and exe $=$ ecxe. Moreover, ef $(c x)=$ $e f(x) \in A$ and $f(c x) e=f(x) c e=f(x) e \in A$. It follows from 2.5 that for any $\lambda \in \mathbf{C} \backslash X_{\eta}, \lambda-z \in \operatorname{Inv}_{0}(\tilde{I})$, where $X_{\eta}=\{\xi: \operatorname{dist}(\xi, s p(c x))<\eta\}$. It then follows 2.6, if $\eta$ is small enough, there are normal elements $\left.y_{1} \in M_{L}(e I e)\right)$ and $\left.y_{2} \in M_{L+1}(e I e)\right)$ with finite spectra contained in $s p(c x)$ such that

$$
\left\|z \oplus y_{1}-y_{2}\right\|<\epsilon / 4
$$

for some integer $L$. Without loss of generality, we may further assume that $y_{1}=$ $\sum_{i=1}^{k} \lambda_{i} d_{i}$, where $d_{i}$ are mutually orthogonal projections in $M_{L}(e I e)$ such that $\sum_{i=1}^{k} d_{i}=$ the identity of $M_{k}(e I e)$.

Now we will "absorb" $y_{1}$. Since $I$ is purely infinite simple $C^{*}$-algebra, there is a partial isometry

$$
v \in(p \oplus e \oplus \cdots \oplus e) M_{L+1}(I)(p \oplus e \oplus \cdots \oplus e)
$$

(there are $L$ copies of $e$ ) such that $v^{*} d_{i} v \leq p_{i}, i=1,2, \ldots, k$,

$$
v^{*} v=\sum_{i=1}^{k} v^{*} d_{i} v \text { and } v v^{*}=e \oplus e \oplus \cdots \oplus e
$$

(there are $L$ copies of $e$ ). There is then a partial isometry $u$ such that

$$
u^{*}\left(z \oplus y_{1}\right) u=\sum_{i=1}^{k} \lambda_{i} q_{i}^{\prime} \oplus z
$$


and $u^{*} y_{2} u$ is normal and has finite spectrum, where $p_{i}^{\prime}=v^{*} d_{i} v$. So

$$
\left\|\sum_{i=1}^{k} \lambda_{i} p_{i} \oplus y-\left[\sum_{i=1}^{n} \lambda_{i}\left(p_{i}-p_{i}^{\prime}\right) \oplus \sum_{j=1}^{n} \alpha_{j}\left(q_{j}-e_{j}\right) \oplus u^{*} y_{2} u\right]\right\|<\epsilon / 2 .
$$

Therefore

$$
\left\|x-\left[\sum_{i=1}^{k} \lambda_{i}\left(p_{i}-p_{i}^{\prime}\right) \oplus \sum_{j=1}^{n} \alpha_{j}\left(q_{j}-e_{j}\right) \oplus u^{*} y_{2} u\right]\right\|<\epsilon
$$

Theorem 2.8. Let $A$ be a $C^{*}$-algebra of real rank zero. Suppose that there is a sequence of ideals:

$$
I_{1} \subset I_{2} \subset \cdots \subset I_{n} \subset I_{n+1} \subset \cdots
$$

such that $A$ is the closure of $\bigcup_{n=1}^{\infty} I_{n}$ and each $I_{n+1} / I_{n}$ is a purely infinite simple $C^{*}$-algebra. Then $A$ has weak (FN).

Proof. Let $x \in A$ be a normal element with $\Gamma(x)=0$, and let $\pi_{n}: A \rightarrow A / I_{n}$ be a quotient map. Since

$$
\begin{gathered}
\operatorname{dist}\left(x, I_{n}\right) \rightarrow 0, \quad \text { as } n \rightarrow \infty, \\
\left\|\pi_{n}(x)\right\| \rightarrow 0 .
\end{gathered}
$$

Let $X_{n}=s p\left(\pi_{n}(x)\right)$. For any $\epsilon>0$, there is an integer $N$ such that if $n>N$,

$$
|\lambda|<\epsilon / 2 \text { for all } \lambda \in X_{n} .
$$

Set a positive continuous function $f$ defined on $s p(x)$ such that $f$ vanishes on $X_{N}$ and $\|f(\xi)-\xi\|<\epsilon / 2$ for all $\xi \in s p(x)$. Then we have

$$
\|f(x)-x\|<\epsilon / 2 .
$$

Clearly, $f(x) \in I_{N}$. So it is enough to prove the theorem for finitely many $I_{n}$ 's. Suppose that $I_{1} \subset I_{2}=A$ and $I_{1}$ and $I_{2} / I_{1}$ are purely infinite simple $C^{*}$-algebras and $A$ has real rank zero. Since purely infinite simple $C^{*}$-algebras have weak (FN) (see $[\operatorname{Ln} 11,4.4]$ ), we can apply 2.7. Suppose that we show that 2.8 holds for $n$ ideals. Now if $I_{1} \subset I_{2} \subset \cdots \subset I_{n+1}=A, I_{k+1} / I_{k}$ is a purely infinite simple $C^{*}$ algebra for each $k$ and $A$ has real rank zero. By induction assumption, $A / I_{1}$ has weak (FN). So 2.7 applies.

Remark 2.9. Let

$$
I \rightarrow A \rightarrow B \rightarrow 0
$$

be a short exact sequence of $C^{*}$-algebras, where $B$ is a Bunce-Deddens algebra and $I$ is isomorphic to $O_{n} \otimes \mathcal{K}$. Since $K_{1}\left(O_{n} \otimes \mathcal{K}\right)=0$, by [Zh4, 3.2], $A$ has real rank zero. It follows from 2.7 that $A$ has weak (FN).

Corollary 2.10. Let $A$ be a $\sigma$-unital purely infinite simple $C^{*}$-algebra with $K_{1}(A)$ $=0$. Then $M(A)$ has weak $(F N)$.

Proof. By $[\mathrm{Zh} 6,1.3], M(A) / A$ is purely infinite simple and $M(A)$ has real rank zero. 
2.11. Let $A$ be a non-elementary separable simple $C^{*}$-algebras with real rank zero and stable rank one. Fix a nonzero projection $e \in A$. Let $\Delta$ be the set of positive homomorphisms $\tau$ from $K_{0}(A)$ into $\mathbf{R}$ such that $\tau(e)=1$ and $Q T(A)$ be the set of (lower semicontinuous) quasitraces $\tau$ such that $\tau(e)=1$. It follows from [BH, III] that there is an (affine) homeomorphism $\chi: Q T(A) \rightarrow \Delta$. We will identify these two compact sets. In fact $\Delta$ is a Choquet simplex ([BH, II. 4.4]). We will use the notation $\operatorname{Aff}(\Delta)$ for the set of all real affine continuous functions defined on $\Delta$. The map $\chi$ gives a homomorphism $\theta: K_{0}(A) \rightarrow A f f(\Delta)$ (see [BH, III. 1.3]). If $K_{0}(A)$ is weakly unperforated, then $K_{0}(A)$ has the strict order induced from $\theta$. Moreover, since $A$ has stable rank one, for any two projections in $M_{\infty}(A)$ if $\tau(p)<\tau(q)$ for all $\tau \in Q T(A)$, then $q \succeq p$, i.e., there is a partial isometry $v \in M_{\infty}(A)$ such that $v^{*} v=p, v v^{*} \leq q$. Furthermore, $\theta\left(K_{0}(A)\right)$ is dense in $\operatorname{Aff}(\Delta) . K_{0}(A)$ is said to have finite rank (countable rank) if there are only finitely (countably) many extreme points in $\Delta$. If $K_{0}(A)$ has rank $n$, then $\operatorname{Aff}(\Delta) \cong \mathbf{R}^{n}$. We say that $x \in K_{0}(A)$ is infinitesimal if $-\epsilon e \leq x \leq \epsilon e$ for all $0<\epsilon \in \mathbf{Q}$ (see [Eff, 4]). If $K_{0}(A)$ is weakly unperforated, by the proof of [Eff, 4.2],

$$
\operatorname{ker} \theta=\left\{x \in K_{0}(A): x \text { is infinitesimal }\right\} .
$$

Let $\hat{1}(\tau)=\tau(1)=\sup \{\tau(p): p \in A\}$. We set

$$
\begin{gathered}
S=\{\tau \in \Delta: \hat{1}(\tau)<\infty\}, \\
J=\left\{f \in \theta\left(K_{0}(A)\right): f(\tau)=0, \text { if } \tau \in S\right\}
\end{gathered}
$$

and

$$
S_{J}=\{\tau \in \Delta: f(\tau)=0 \text {, if } f \in J\} .
$$

Then $S_{J}$ is closed and $S \subset S_{J}$. One can easily check that $S_{J}$ is a face of $\Delta$. In [Ln10], we show that if $A$ is a nonunital separable simple $C^{*}$-algebra with real rank zero, stable rank one and weakly unperforated $K_{0}(A)$, then

(1) $K_{1}(M(A))=\{0\}$ (this holds without assuming that $K_{0}(A)$ is weakly unperforated);

(2) $U(M(A) / A) / U_{0}(M(A) / A)=K_{1}(M(A) / A)=\theta^{-1}(J)$;

Notice that (2) implies that $\operatorname{ker} \theta \subset K_{1}(M(A) / A)$. If the function $\hat{1}(\tau)=\tau(1)$ is continuous on $\Delta$, then we say that $A$ has continuous scale (see [Ln10] and [Ln1]). If $A$ has continuous scale, then $K_{1}(M(A) / A)=\operatorname{ker} \theta$.

The notations and results stated here will be used frequently without warning.

Proposition 2.12. Let $A$ be a separable simple $C^{*}$-algebra with real rank zero, stable rank one, trivial $K_{1}(A)$ and weakly unperforated $K_{0}(A)$ of rank $n$. Then $M(A) / A$ has a (finite) sequence of ideals

$$
0=I_{0} \subset I_{1} \subset \cdots \subset I_{k} \subset J_{k+1}=M(A) / A \quad(k \leq n)
$$

such that $I_{i+1} / I_{i}$ is purely infinite simple for each $i$.

Proof. Fix a nonzero projection $e \in A$. Let $\tau_{1}, \tau_{2}, \ldots, \tau_{n}$ be extreme points of

$$
\Delta=\{\tau: \tau(e)=1\},
$$

where $\tau$ are quasitraces on $A$. Suppose that $\tau_{i}(1)=\infty, i=1,2, \ldots, k$. Set

$$
J_{i}^{+}=\left\{a \in M(A)_{+}: \tau_{m}(a)<\infty, m=1,2, \ldots, k-i+1\right\}, \quad i=1,2, \ldots, k+1 .
$$


Let $J_{i}$ be the ideal generated by $J_{i}^{+}$. It is enough to show that $J_{i+1} / J_{i}$ is purely infinite and simple. We also let $J_{0}=A$. The fact that we have finite tower $A=$ $I_{0} \subset J_{1} \subset \cdots \subset J_{k+1}=M(A)$ and $J_{i} / J_{i-1}$ is simple follows from the proof of Theorem 2 in [Ln1]. We now use induction on $k$. If $k=0$, then $A$ is finite, it follows from [Ln1] and [Zh2] that $M(A) / A$ is purely infinite simple. Suppose that 2.12 holds for $k$. Note, by [Ln4, 2.8] (see also [Ln7, 9]), $K_{1}\left(J_{i-1}\right)=0$. So every projection in $J_{i} / J_{i-1}$ lifts to a projection in $J_{i}$. Let $e \in J_{i} / J_{i-1}$ be a projection. Then there is a projection $E \in M(A)$ such that $\phi_{i}(E)=e$, where $\phi_{i}: J_{i} / J_{i-1}$ is the quotient map. Thus, by induction assumption, $e J_{i} / J_{i-1} e$ is a purely infinite simple $C^{*}$-algebra for $i=1,2, \ldots, k$. Since $\theta\left(K_{0}(A)\right)$ is dense in $\mathbf{R}^{n}$, it is easy to see that there are projections $p_{i} \in J_{i} \backslash J_{i-1}, i=2,3, \ldots, k$. So, there is at least one nonzero $e \in J_{i} / J_{i-1}$. Since $J_{i} / J_{i-1}$ is simple and has nonzero projections, it is the linear span of projections $([\mathrm{Pd} 3])$. It follows [Zh7, 1.1] that $\left(J_{i} / J_{i-1}\right) \otimes \mathcal{K}$ has an approximate identity consisting of projections. Thus $\left(J_{i} / J_{i-1}\right) \otimes \mathcal{K}$ is a purely infinite simple $C^{*}$-algebra for $i=1,2, \ldots, k$, whence $J_{i} / J_{i-1}$ is a purely infinite simple $C^{*}$-algebra for $i=1,2, \ldots, k$.

It remains to show that $M(A) / J_{k}$ is purely infinite and simple. It suffices to show that the closure of $\phi_{k+1}(x)\left(M(A) / J_{k}\right) \phi_{k+1}(x)$ has an infinite projection for every positive element $x$ in $M(A) / A$. By [Zh6, 1.1], the closure $x(M(A) / A) x$ is the closure of linear combinations of projections in the closure $x(M(A) / A) x$, which implies that at leat one projection $p$ in the closure $x(M(A) / A) x$ such that $\phi_{k+1}(p)$ is not zero. It is easy to find a projection $q \leq p$ such that $\tau_{i}(p-q)=\infty$ and $\tau_{i}(q)=\infty$ for $i=1,2, \ldots, k$. In particular $\phi_{k}(q) \neq 0$ and $\phi_{k}(p-q) \neq 0$. By $[\operatorname{Ln} 10$, $3]$, one shows that $\phi_{k}(p)$ is equivalent to $\phi_{k}(q)$. This implies that $\phi_{k}(p)$ is infinite.

Corollary 2.13. Let $A$ be a separable simple $C^{*}$-algebra with real rank zero, stable rank one, trivial $K_{1}(A)$ and weakly unperforated $K_{0}(A)$ of finite rank. Then $M(A) / A$ has weak $(F N)$.

Proof. As in the proof of 2.12, $K_{1}\left(J_{i}\right)=0$ for each $J_{i}$. Then, by repeated application of [Zh2, 2.3] (also [BP, 3.14]), $M(A)$ has real rank zero. Then 2.13 follows from $2.12,2.8$ and [Ln11] immediately.

\section{The generalized Berg theOrem}

Definition 3.1 (cf. [Zh1, 1.3] and [LS]). Let $A$ be a $C^{*}$-algebra. We say that the Weyl-von Neumann-Berg theorem holds for $A$ if every normal element of $M(A)$ is quasidiagonal; i.e. given any normal element $x \in M(A)$ there is an approximate identity $\left\{e_{n}\right\}$ for $A$ consisting of projections such that

$$
x-\sum_{n=1}^{\infty}\left(e_{n}-e_{n-1}\right) x\left(e_{n}-e_{n-1}\right) \in A,
$$

where the sum is taken in the strict topology.

Loring and Spielberg [LS] show that a normal element $x \in M(A)$ may not be quasidiagonalized. It happens when the normal element $x$ has $\gamma(x) \neq 0$. Suppose that $A$ is a separable simple $A F$-algebra with finitely many extreme traces. We will show that a normal element $x \in M(A)$ is quasidiagonal if and only if $\gamma(x)=0$.

Lemma 3.2 (cf. [Zh1, 3.9]). Let $A$ be a $\sigma$-unital $C^{*}$-algebra. Suppose that $M(A)$ has real rank zero. If $y \in M(A), \pi(y)$ is a normal element in $M(A) / A$ and $\pi(y)$ can be approximated by normal elements in $M(A) / A$ with finite spectra, then, for 
any $\epsilon>0$, there is an approximate identity $\left\{e_{n}\right\}$ for $A$ consisting of projections and $a \in A$ such that

$$
y=\sum_{n=1}^{\infty}\left(e_{n}-e_{n-1}\right) y\left(e_{n}-e_{n-1}\right)+a,
$$

$\|a\|<\epsilon / 2$, and

$$
\left\|\left(e_{n}-e_{n-1}\right) y-y\left(e_{n}-e_{n-1}\right)\right\|<\epsilon / 2^{n} .
$$

Proof. The proof is contained in [Zh1, 3.9]. For any $\epsilon>0$, there exist mutually orthogonal projections $q_{1}, q_{2}, \ldots, q_{n} \in M(A)$ and $\lambda_{1}, \lambda_{2}, \ldots, \lambda_{n} \in s p(y)$ such that

$$
\left\|\pi(y)-\sum_{i=1}^{n} \lambda_{i} q_{i}\right\|<\epsilon
$$

Then, since $A$ has real rank zero, each $q_{i}=\sum_{j=1}^{\infty} e_{i j}$, where $e_{i j}$ are mutually orthogonal projections in $q_{i} A q_{i}$ and the sum converges in the strict topology. So we may write

$$
\sum_{i=1}^{n} \lambda_{i} q_{i}=\sum_{m=1}^{\infty} \alpha_{m} p_{m}
$$

where $\left\{p_{m}\right\}$ are mutually orthogonal projections in $A$ and $\sum_{m=1}^{\infty} p_{m}$ converges to the identity 1 in the strict topology. Then we can apply [Zh1, 3.9] ((b) implies (e)). To have exactly the same inequalities required in the lemma, one can take a subsequence of $\left\{e_{n}\right\}$ which is still an approximate identity.

Theorem 3.3. Let $A$ be a separable simple $C^{*}$-algebra with real rank zero, stable rank one, trivial $K_{1}(A)$, weakly unperforated $K_{0}(A)$ of finite rank. Suppose that every quasitrace is a trace. Then a normal element $x \in M(A)$ is quasidiagonal if and only if $\gamma(x)=0$.

Proof. Since $x$ is normal, it follows from $[\operatorname{Ln} 13,1.13]$ that $\gamma^{I}(x)=0$ for every ideal $I$ of $M(A)$ which contains $A$ but is not $A$ itself.

If $\gamma(x)=0$, then $\Gamma(\pi(x))=0$. Since $M(A) / A$ has weak (FN), by $2.13, \pi(x)$ can be approximated by normal elements (in $M(A) / A$ ) with finite spectrum. Then we can apply 3.2 .

Now we suppose that $x$ is quasidiagonal. We will show that $\gamma(x)=0$. Let $\left\{e_{n}\right\}$ be an approximate identity for $A$ consisting of projections such that

$$
x-\sum_{n=1}^{\infty}\left(e_{n}-e_{n-1}\right) x\left(e_{n}-e_{n-1}\right) \in A .
$$

Let $f \in C(\operatorname{sp}(x))$ such that $\pi(f(x))$ is a unitary in $M(A) / A$. Since $\left\{e_{n}\right\}$ is an approximate identity for $A$,

$$
\left\|\left(e_{n}-e_{n-1}\right) x-x\left(e_{n}-e_{n-1}\right)\right\| \rightarrow 0 \text { as } n \rightarrow \infty .
$$

It follows that

$$
\pi\left[\sum_{n=1}^{\infty}\left(e_{n}-e_{n-1}\right) f(x)\left(e_{n}-e_{n-1}\right)\right]=\pi(f(x))
$$

Therefore

$$
\left\|\left(e_{n}-e_{n-1}\right)-\left(e_{n}-e_{n-1}\right) f(x)\left(e_{n}-e_{n-1}\right)^{*}\left(e_{n}-e_{n-1}\right) f(x)\left(e_{n}-e_{n-1}\right)\right\| \rightarrow 0
$$


and

$$
\left\|\left(e_{n}-e_{n-1}\right)-\left(e_{n}-e_{n-1}\right) f(u)\left(e_{n}-e_{n-1}\right)\left(e_{n}-e_{n-1}\right) f(x)\left(e_{n}-e_{n-1}\right)^{*}\right\| \rightarrow 0
$$

as $n \rightarrow \infty$. Thus, by a standard argument involving the polar decomposition, there are unitaries $u_{n} \in\left(e_{n}-e_{n-1}\right) A\left(e_{n}-e_{n-1}\right)$ such that

$$
\left\|u_{n}-\left(e_{n}-e_{n-1}\right) f(x)\left(e_{n}-e_{n-1}\right)\right\| \rightarrow 0
$$

as $n \rightarrow \infty$. Notice that

Therefore

$$
\sum_{n=1}^{\infty}\left(e_{n}-e_{n-1}\right) f(x)\left(e_{n}-e_{n-1}\right)-\sum_{n=1}^{\infty} u_{n} \in A .
$$

$$
\pi\left(\sum_{n=1}^{\infty} u_{n}\right)=\pi(f(u)) .
$$

Moreover, $\sum_{n=1}^{\infty} u_{n}$ is a unitary in $M(A)$. It follows from [Ln7, 9] that $K_{1}(M(A))=$ 0 . This implies that $\gamma(x)=0$.

Lemma 3.4. Let $A$ be as in 3.2. If $x \in M(A)$ is a normal element, then im $[\gamma(\pi(x))]$ $\subset \operatorname{ker} \theta\left(K_{0}(A)\right)$.

Proof. Let $f: s p(\pi(x)) \rightarrow S^{1}$ be a continuous function. Then $f(\pi(x))$ is a unitary in $M(A) / A$. So there is a partial isometry $v \in M(A)$ such that $\pi(v)=f(\pi(x))$. Let $g \in C(s p(x))$ such that $\pi(g(x))=f(\pi(x))$. Set $y=g(x)$. Then there is an element $a \in A$ such that $v=y+a$. Let $\left\{e_{n}\right\}$ be an approximate identity for $A$. Then $a^{*} y=\lim _{n \rightarrow \infty} a^{*} e_{n} y$ and $a y^{*}=\lim _{n \rightarrow \infty} a\left(e_{n} y\right)^{*}$. Therefore, for any trace $\tau$, $\tau\left(a^{*} y\right)=\tau\left(a y^{*}\right)$. Similarly, $\tau\left(y^{*} a\right)=\tau\left(y a^{*}\right)$. Since $y^{*} y=y y^{*}$, we have

$$
\tau\left(v^{*} v-v v^{*}\right)=\tau\left(a^{*} a-a a^{*}\right)+\tau\left(a^{*} y-a y^{*}\right)+\tau\left(y^{*} a-y a^{*}\right)=0 .
$$

This implies that $\left[v^{*} v\right]-\left[v v^{*}\right] \in \operatorname{ker} \theta\left(K_{0}(A)\right)$. It follows from $[\operatorname{Ln} 7,9]$ that $K_{1}(M(A))$ $=0$. From the six-term exact sequence in $K$-theory, the map

$$
K_{1}(M(A) / A) \rightarrow K_{0}(A)
$$

is injective. Therefore $\operatorname{im}[\gamma(x)] \subset \operatorname{ker} \theta\left(K_{0}(A)\right)$.

Corollary 3.5. Let $A$ be as in 3.3. Suppose that $K_{0}(A)$ has no infinitesimal elements (i.e. $\operatorname{ker} \theta=0$ ). Then every normal element $x \in M(A)$ is quasidiagonal.

We would now like to present a stronger version of the Berg theorem for separable simple $A F$-algebras with continuous scale and $K_{0}(A)$ of countable rank.

The following lemma is proved in [EGLP, 4.4], even though it stated differently.

Lemma 3.6 (cf. [EGLP, 4.4], [Ln11, 4.8], and [Ln6, 2]). Let $A$ be a unital $C^{*}$ algebra of real rank zero. Let $\Delta$ be a set of countably many (finite) traces on A. Suppose that $\Delta$ is $w^{*}$-compact and the identity of $A$ is a continuous function on $\Delta$. Suppose that $\phi: C(X) \rightarrow A$ is a monomorphism, where $X$ is a compact metric space. For any $\epsilon>0$, any finitely many $f_{1}, f_{2}, \ldots, f_{m} \in C(X)$, and integer $K>0$ there are mutually orthogonal projections $p_{1}, p_{2}, \ldots, p_{n}$ in $A$ and $\lambda_{1}, \lambda_{2}, \ldots, \lambda_{n} \in X$ such that

$$
\left\|\phi\left(f_{s}\right)-\left(y_{s}+\sum_{i=1}^{n} f_{s}\left(\lambda_{i}\right) p_{i}\right)\right\|<\epsilon, \quad s=1,2, \ldots, m
$$


where $y_{s}=\left(1-\sum_{i=1}^{n} p_{i}\right) \phi\left(f_{s}\right)\left(1-\sum_{i=1}^{n} p_{i}\right)$

$$
\begin{gathered}
\left\|\left(1-\sum_{i=1}^{n} p_{i}\right) \phi\left(f_{s}\right)-\phi\left(f_{s}\right)\left(1-\sum_{i=1}^{n} p_{i}\right)\right\|<\epsilon, \\
t\left(p_{k}\right)>K t\left(1-\sum_{i=1}^{n} p_{i}\right)
\end{gathered}
$$

for all $t \in \Delta, s=1,2, \ldots, m, k=1,2, \ldots, n, j=1,2, \ldots, l$, and for any $\lambda \in X$, there is $\lambda_{i}$ such that

$$
\operatorname{dist}\left(\lambda, \lambda_{i}\right)<\epsilon
$$

Theorem 3.7. Let $A$ be a separable simple $C^{*}$-algebra with real rank zero, stable rank one and continuous scale. Suppose that $K_{1}(A)=0$, every quasitrace of $A$ is a trace and $K_{0}(A)$ has countable rank. Then every normal element $x \in M(A)$ with $\gamma(x)=0$, and $\epsilon>0$, there exist an approximate identity $\left\{e_{n}\right\}$ for $A$ consisting of projections and $a \in A$ such that

$$
x=\sum_{n=1}^{\infty} \lambda_{n}\left(e_{n}-e_{n-1}\right)+a
$$

where $\left\{\lambda_{n}\right\}$ is a bounded sequence of complex numbers and $\|a\|<\epsilon$.

Proof. Since $A$ has continuous scale, by [Ln2], $M(A) / A$ is simple, and hence purely infinite simple $([\mathrm{Zh} 2,5.1])$. So $M(A)$ and $M(A) / A$ has real rank zero. Let $\Delta$ be as in 2.11. It follows from 3.6 that for any $\eta>0$ and integer $L>0$, there are mutually orthogonal projections $p_{i}^{\prime} \in M(A)$ and $\lambda_{i} \in \operatorname{sp}(x)$ such that

$$
\left\|x-\sum_{i=1}^{n} \lambda_{i} p_{i}^{\prime}-p^{\prime} x p^{\prime}\right\|<\eta / 4
$$

and

$$
t\left(p_{i}^{\prime}\right)>(L+1) t\left(p^{\prime}\right)
$$

for all $i$ and $t \in \Delta$, where $p^{\prime}=1-\sum_{i=1}^{n} p_{i}^{\prime}$ and $\left\{\lambda_{i}\right\}$ is $\eta / 16$-dense in $s p(x)$. Since $A$ has real rank zero and $p_{i}^{\prime} A p_{i}^{\prime}$ has continuous scale for each $i$, there is a projection $p_{i} \in p_{i}^{\prime} A p_{i}^{\prime}$ for each $i$ such that

$$
t\left(p_{i}\right)>(L+1) t\left(p^{\prime}+\sum_{i=1}^{n}\left(p_{i}^{\prime}-p_{i}\right)\right)
$$

for each $i$ and $t \in \Delta$. From the proof of $[\operatorname{Ln} 11,3.4]$, we have

$$
\left\|x-\sum_{i=1}^{n} \lambda_{i} p_{i}-p x p\right\|<\eta / 4,
$$

where $p=1-\sum_{i=1}^{n} p_{i}=p^{\prime}+\sum_{i=1}^{n}\left(p_{i}^{\prime}-p_{i}\right)$. Set $z=p x p$. Then $\pi(x)=\pi(z)$. It follows from $[\operatorname{Ln} 12,7.1]$ that there is an element $a \in p A p$ such that

$$
z=\sum_{k=1}^{\infty} \lambda_{n}\left(q_{n}-q_{n-1}\right)+a
$$


where $\lambda_{n} \in s p(x)$ and $\left\{q_{n}\right\}$ is an approximate identity for $p A p$ consisting of projections. There is an integer $N$ such that

$$
\left\|\left(1-q_{N}\right) a\right\|<\eta / 8 \text { and } \|\left(a\left(1-q_{N}\right) \|<\eta / 8 .\right.
$$

We may write

$$
z=\sum_{n=N+1}^{\infty} \lambda_{n}\left(q_{n}-q_{n-1}\right)+q_{N} z q_{N}+b
$$

where $b \in A$ and $\|b\|<\eta / 4$. We also have

$$
\left\|q_{N} z-z q_{N}\right\|<\eta / 4 \text {. }
$$

Notice that $z=p x p$. So $q_{N} z q_{N}=q_{N} x q_{N}$. By Lemma 2.6, if $\eta$ is small enough, there are normal elements $z_{1} \in M_{L}\left(q_{N} A q_{N}\right)$ and $z_{2} \in M_{L+1}\left(q_{N} A q_{N}\right)$ with finite spectra $s p\left(z_{i}\right) \in s p(x)$ such that

$$
\left\|q_{N} z q_{N} \oplus z_{1}-z_{2}\right\|<\epsilon / 8 \text {. }
$$

Notice that $L$ depends only in $\epsilon$. We may also assume that $z_{1}=\sum_{i=1}^{n} \lambda_{i} d_{i}$ for some mutually orthogonal projections $d_{i} \in M_{L}\left(q_{N} A q_{N}\right)$. Since $t\left(p_{i}\right)>t\left(d_{i}\right)$ for all $t \in \Delta$ and $i=1,2, \ldots, n$, There is a unitary $\left.u \in M_{L+1}\left((1-p)+q_{N}\right) A(1-p)+q_{N}\right)$ such that

$$
\left\|\sum_{i=1}^{n} \lambda_{i} p_{i}+q_{N} z q_{N}-U^{*} z_{2} U\right\|<\epsilon / 8
$$

Furthermore, $z_{3}=U^{*} z_{2} U \in\left((1-p)+q_{N}\right) A\left((1-p)+q_{N}\right)$ and $s p\left(z_{3}\right)$ is finite. If $\eta$ is small enough, we then have

$$
x=\sum_{n=N+1}^{\infty} \lambda_{n}\left(q_{n}-q_{n-1}\right)+z_{3}+b_{1}+b,
$$

where $b_{1} \in A$ and $\left\|b_{1}+b\right\|<\epsilon$.

Corollary 3.8. Theorem 3.7 holds for $\sigma$-unital purely infinite simple $C^{*}$-algebras with trivial $K_{1}$-group.

Proof. The proof is the same as that of 3.7. Notice that in a purely infinite simple $C^{*}$-algebra $A$ any projection is "larger" than any others and in $M(A) \backslash A$ any two projections are equivalent.

\section{The Generalized BDF-Theorem}

In this section, $A$ is always a separable simple $C^{*}$-algebra with real rank zero, stable rank one, trivial $K_{1}(A)$ and weakly unperforated $K_{0}(A)$ of finite rank. Furthermore, we assume that all quasitraces are traces.

4.1. After we discussed the Berg theorem, it would be unnatural not to consider the BDF-theorem for essentially normal elements. Essentially normal elements are those elements $x \in M(A)$ such that $\pi(x)$ is normal. The question, as in the operator theory, is when can an essentially normal element $x \in M(A)$ be written as $x=y+a$, where $y \in M(A)$ is normal and $a \in A$ ? A natural first thought might be : "index must be zero." Well, by 3.7, $\gamma_{I}(x)=0$ for any ideal $I$ of $M(A)$ which strictly contains $A$, if $x$ is a normal element in $M(A)$. How about the Fredholm index? Mingo and Spielberg's examples show that even normal elements can have 
nonzero Fredholm index. So, the second thought is that it would be hopeless to give a definite answer. However, recent results in extension theory [Ln12] encourage us to have the third thought. Lemma 3.4 said that normal elements can only have an infinitesimal nonzero Fredholm index. We discover, by using results in [Ln12], that an essentially normal element $x \in M(A)$ with infinitesimal $\Gamma(x)$ has form $y+a$.

Lemma $4.2([\operatorname{Ln} 14,3])$. Let $p$ and $q$ be two open projections in $A^{* *}$ both not in $A$. If $t(p)=t(q)$ for all $t \in \Delta$, where $\Delta$ is the same as in 2.8, then $\operatorname{Her}(p) \cong \operatorname{Her}(q)$, where $\operatorname{Her}(p)$ and $\operatorname{Her}(q)$ are hereditary $C^{*}$-subalgebras corresponding to the open projection $p$ and $q$, respectively.

Proof. The proof is exactly the same as that of $[\operatorname{Ln} 14,3]$.

Lemma 4.3 (cf. $[\mathrm{MS}])$. For any $\zeta \in \operatorname{ker} \theta$, there is a normal element $x \in M(A)$ such that $s p(x)=\bar{D}, s p(\pi(x))=S^{1}$ and $[\pi(x)]=\zeta$ in $K_{1}(M(A) / A)$.

Proof. Take a nonzero projection $e \in A$. It follows from [EL, 7.3] that there is a homomorphism $\phi: C\left(S^{2}\right) \rightarrow e A e$ such that $\phi_{*}(b)=\zeta$, where $b$ is the "Bott" element. Let us denote the restriction of $\phi$ on $C_{0}(D)$ by $\phi$ itself. Then, as in [MS], $\phi_{*}(b)=\zeta$. Let $h$ be a strict positive element of $C_{0}(D)$ and $B=\operatorname{Her}(\phi(h))$, the hereditary $C^{*}$-subalgebra of $e A e$ generated by $\phi(h)$. Combining [MS, Proposition] and [MS, Theorem 2], we obtain a normal element $y \in M(B)$ such that $s p(y)=\bar{D}$ and $[\pi(y)]=\zeta$ in $K_{1}(M(B) / B)$.

Since $\theta\left(K_{0}(A)\right)$ is dense in $\mathbf{R}^{n}$ (see 2.11), there is a projection $p \in M(A)$ such that $t(p)=t(q)$ for all $t \in \Delta$, where $q$ is the open projection in $A^{* *}$ corresponding to $B$. It follows from 4.2 that $B \cong p A p$. Therefore $M(B) \cong p M(A) p$. We may assume that $y \in p M(A) p$. Let 1 be the identity of $M(A)$. Set $x=y \oplus u$, where $u$ is a unitary in $(1-p) M(A)(1-p)$. Notice that $K_{1}((1-p) M(A)(1-p))=0$. So $s p(x)=\bar{D}$ and

$$
[\pi(x)]=\zeta \text { in } K_{1}((M(A) / A)
$$

Lemma 4.4. Let $X$ be a compact subset of the closed unit disk $\bar{D}$, and $O_{1}, O_{2}, \ldots$, $O_{n}, \ldots$ be the sequence of bounded components of $\mathbf{C} \backslash X$. Set

$$
X_{i}=\left\{\lambda_{i}-\xi: \xi \in X\right\}
$$

for some $\lambda_{i} \in O_{i}$. Let $g_{i}: X_{i} \rightarrow S^{1}$ be a generator of $K_{1}(C(X))$ corresponding to $O_{i}$. If $\zeta \in \operatorname{ker} \theta$, then for each $i$, there is a normal element $x_{i} \in M(A)$ such that $s p\left(x_{i}\right) \subset \bar{D}, s p\left(\pi\left(x_{i}\right)\right) \subset X$ and

$$
\left[g_{i}\left(\lambda_{i}-\pi\left(x_{i}\right)\right)\right]=\zeta \text { and }\left[g_{j}\left(\lambda_{j}-\pi\left(x_{i}\right)\right)\right]=0
$$

in $K_{1}(M(A) / A)$ if $j \neq i$.

Proof. Notice that we identify $S^{1}$ with the unit circle on the plane. There is a compact subset $F_{i} \subset X_{i}$ such that $g_{i}: F_{i} \rightarrow S^{1}$ is a homeomorphism. Let $x \in M(A)$ be the normal element in 4.3. There is $f_{i} \in C(\operatorname{sp}(x))$ such that $\left\|\lambda_{i}-f_{i}\right\| \leq 1$ and

$$
\pi\left(f_{i}(x)\right)=g_{i}^{-1}(\pi(x)) .
$$

Define $x_{i}=\lambda_{i}-f_{i}(x)$. 
Lemma 4.5. Let $X$ be a compact subset of the plane, and let $\psi: K_{1}(C(X)) \rightarrow$ ker $\theta$ be a homomorphism. Then there is a normal element $x \in M(A)$ satisfying the following:

(1) $s p(\pi(x))=X$,

(2) $\phi_{*}=\psi$, where $\phi: s p(\pi(x)) \rightarrow M(A) / A$ is the isomorphism induced by the element $\pi(x)$.

Proof. Without loss of generality, we may assume that $X \subset \bar{D}$. It is easy to construct a sequence of mutually orthogonal projections $p_{i} \in M(A) \backslash A$ such that $\sum_{i=1}^{\infty} p_{n}$ converges to a projection $p$ in the strict topology, where $p \in M(A)$ and $1-p \notin A$. For each $i$, by applying 4.3 , there is $x_{i} \in p_{i} M(A) p_{i}$ satisfying

$$
\left[g_{i}\left(\lambda_{i}-x_{i}\right)\right]=\psi\left(g_{i}^{\prime}\right) \text { and }\left[g_{j}\left(\lambda_{j}-x_{i}\right)\right]=0
$$

for $j \neq i$ and $g_{i}^{\prime}(z)=g_{i}\left(\lambda_{i}-z\right)$. Set

$$
y=\sum_{i=1}^{\infty} x_{i}
$$

Note that $\sum_{i=1}^{n} x_{i}$ converges to $y$ in the strict topology. Then $y$ is a normal element in $M(A)$. Take a dense sequence of numbers in $X$ such that each isolated point occurs infinitely often and an approximate identity $\left\{e_{n}\right\}$ for $(1-p) A(1-p)$ consisting of projections. Set

$$
z=\sum_{n=1}^{\infty} \lambda_{n}\left(e_{n}-e_{n-1}\right)
$$

Define $x=y+z$. It is easy to see that the element $x$ satisfies the required conditions.

The following result is basically contained in [Ln12].

Lemma 4.6. Let $A$ be a separable simple $C^{*}$-algebra with real rank zero, stable rank one, $K_{1}(A)=0$, weakly unperforated $K_{0}(A)$, and let $\sigma: C(X) \rightarrow M(A) / A$ be an extension, where $X$ is a compact metric space. Suppose that $p \in M(A)$ is a projection such that $[\pi(p)]=0$, i.e., $\tau(p) \in \theta\left(K_{0}(A)\right)$ for all $\tau \in \Delta$, and $\sigma_{0}: C(F) \rightarrow M(p A p) / p A p$ is a null extension (see $[\operatorname{Ln} 12,2.2]$ or $\left.[\operatorname{Ln} 12,2.9]\right)$, where $F$ is a compact subset of $X$. Then $\sigma \oplus \sigma_{0}$ is unitarily equivalent to $\sigma$.

Proof. By $[\operatorname{Ln} 12,1.5]$, there is a nonzero projection $q \in M(A)$ and injective maps

$$
\sigma^{\prime}: C(X) \rightarrow M((1-q) A(1-p)) /(1-q) A(1-q)
$$

and $\sigma_{1}: C(F) \rightarrow M(q A q) / q A q$ such that $\sigma_{1}$ is totally trivial and $\sigma=\sigma^{\prime}+\sigma_{1}$. Since $\sigma_{1}$ is totally trivial, there exist an approximate identity $\left\{e_{n}\right\}$ for $q A q$ consisting of projections and a dense sequence $\left\{\xi_{n}\right\}$ in $F$ with isolated points repeated infinitely often such that

$$
\sigma_{1}(f)=\pi\left[\sum_{n=1}^{\infty} f\left(\xi_{n}\right)\left(e_{n}-e_{n-1}\right)\right]
$$

One can find nonzero projections $d_{n} \leq\left(e_{n}-e_{n-1}\right)$ such that $\sum_{n=1}^{\infty} \tau\left(d_{n}\right)$ converges uniformly on $\Delta$. Let $q^{\prime}=\sum_{n=1}^{\infty} d_{n}$ Then $q^{\prime} A q^{\prime}$ has continuous scale. Furthermore 
the map

$$
\sigma_{1}^{\prime}(f)=\pi\left[\sum_{n=1}^{\infty} f\left(\xi_{n}\right)\left(e_{n}-e_{n-1}\right)\right]
$$

is an extension of $C(F)$ by $q^{\prime} A q^{\prime}$. Fix any point $\xi \in F$. Let $h \in C(F)$ be such that $h(\xi)=0$ and $h>0$ elsewhere on $F$. Denote by $B$ the hereditary $C^{*}$-subalgebra of $M\left(q^{\prime} A q^{\prime}\right) / q^{\prime} A q^{\prime}$ generated by $\sigma_{1}^{\prime}(h)$. Then $B$ is $\sigma$-unital. It follows from $[\mathrm{Pd} 2,15]$ that $B^{\perp}$ is a nonzero hereditary $C^{*}$-subalgebra of $M\left(q^{\prime} A q^{\prime}\right) / q^{\prime} A q^{\prime}$. Since $M\left(q^{\prime} A q^{\prime}\right) / q^{\prime} A q^{\prime}$ has real rank zero $([\operatorname{Ln} 7])$, by $[\mathrm{BP}, 2.6], B^{\perp}$ has real rank zero. Therefore there is a nonzero projection $d \in B^{\perp}$. It is easy to verify that $d$ commutes with $i m \sigma_{1}^{\prime}$. Moreover,

$$
\sigma_{1}^{\prime}(f)=f(\xi) d+(1-d) \sigma_{1}^{\prime}(f)(1-d)
$$

for all $f \in C(F)$. Since $M\left(q^{\prime} A q^{\prime}\right) / q^{\prime} A q^{\prime}$ is simple ([Ln2]), it is in fact purely infinite simple. There is a nonzero projection $d^{\prime} \leq d$ such that $\left[\pi\left(q^{\prime}\right)-d^{\prime}\right]=0$ in $K_{0}(M(A) / A)$. Let $p^{\prime} \in M(A)$ be a projection such that $\pi\left(p^{\prime}\right)=\pi\left(q^{\prime}\right)-d^{\prime}$. Set

$$
\sigma_{2}(f)=f(\xi) d^{\prime}+(1-d) \sigma_{1}^{\prime}(f)(1-d)
$$

for all $f \in C(F)$. Then $\sigma_{2}: C(F) \rightarrow M\left(p^{\prime} A p^{\prime}\right) / p^{\prime} A p^{\prime}$ is a (totally trivial) extension. Clearly $p^{\prime}$ commutes with $i m \sigma$. Let $\sigma^{\prime \prime}=p^{\prime} \sigma$. Then $\sigma=\sigma^{\prime \prime} \oplus \sigma_{2}$. It follows from $[\operatorname{Ln} 12,2.3]$ that $\sigma_{2} \oplus \sigma_{0}$ is unitarily equivalent to $\sigma_{2}$. Therefore, $\sigma \oplus \sigma_{0}$ is equivalent to $\sigma$.

Theorem 4.7. Let $x \in M(A)$ be an essentially normal element, i.e., $\pi(x)$ is normal in $M(A) / A$. Then $x$ can be written as $x=y+a$, where $y \in M(A)$ is a normal element and $a \in A$ if and only if $\gamma(\pi(x)) \in \operatorname{ker} \theta$ and $\gamma_{I}(x)=0$ for all ideals $I$ of $M(A)$ which strictly contain $A$.

Proof. The "only if" part follows from 3.6 and [Ln13, 1.13].

Suppose now that $x \in M(A), \pi(x)$ is normal, $\gamma(\pi(x)) \in \operatorname{ker} \theta$ and $\gamma_{I}(x)=0$ for all ideals $I$ which strictly contain $A$. Suppose that $X=\operatorname{sp}(\pi(x))$. Let $\sigma: C(X) \rightarrow$ $M(A) / A$ be the extension induced by the normal element $\pi(x)$. Let $p \in M(A)$ such that $[\pi(p)]=0$, i.e., $\tau(p) \in \theta\left(K_{0}(A)\right)$ for all $\tau \in \Delta$. So $p A p$ has continuous scale and $K_{1}(M(p A p) / p A p)=\operatorname{ker} \theta(\operatorname{see}[\operatorname{Ln} 10])$. By $[\operatorname{Ln} 12,7.1]$, there is an extension $\sigma_{1}: C(X) \rightarrow M(p A p) / p A p$ such that $\gamma_{1}\left(\sigma_{1}\right)=-\gamma(\pi(x))$. Set $B=$ $(1 \oplus p) M_{2}(A)(1 \oplus p)$. Let $z(\xi)=\xi$ for $\xi \in X$. Then $\Gamma\left(\pi(x) \oplus \sigma_{1}(z)\right)=0$. Therefore, by 3.5 , there is a normal element $y_{1} \in M(A)$ such that $\pi\left(y_{1}\right)=\pi(x) \oplus \sigma_{1}(z)$. Now we use the notation $y_{2}$ for a normal element in $M(p A p)$ satisfying the conditions in 4.5, with $\psi=\gamma(\pi(x))$. Let $\sigma_{2}: C(X) \rightarrow M(p A p) / p A p$ be the extension induced by the normal element $\pi\left(y_{2}\right)$. Then $\gamma_{1}\left(\sigma_{1} \oplus \sigma_{2}\right)=0$. By $[\operatorname{Ln} 12,7.1], \sigma_{1} \oplus \sigma_{2}$ is totally trivial. It follows from $[\mathrm{Ln} 12,4.3]$ that there is a totally trivial extension $\sigma_{3}: C(X) \rightarrow M(p A p) / p A p$ such that $\sigma_{1} \oplus \sigma_{2} \oplus \sigma_{3}$ is a null extension. It follows from 4.4 that $\sigma$ is unitarily equivalent to $\sigma \oplus \sigma_{1} \oplus \sigma_{2} \oplus \sigma_{3}$. There is a (diagonal) normal element $y_{3} \in M(A)$ such that $\pi\left(y_{3}\right)=\sigma_{3}(z)$. Since

$$
\sigma \oplus \sigma_{1} \oplus \sigma_{2} \oplus \sigma_{3}(z)=\pi\left(y_{1}\right) \oplus \pi\left(y_{2}\right) \oplus \pi\left(y_{3}\right)
$$

there is a partial isometry $u \in M_{2}(A)$ such that $u^{*} u=1, u u^{*}=1 \oplus p \oplus p \oplus p$ and

$$
\pi\left(u^{*} x u\right)=\pi\left(y_{1}\right) \oplus \pi\left(y_{2}\right) \oplus \pi\left(y_{3}\right) .
$$

We conclude that $x=y+a$, where $y=u\left(y_{1} \oplus y_{2} \oplus y_{3}\right) u^{*}$ and $a \in A$. 
Corollary 4.8. Suppose that $K_{0}(A)$ has no infinitesimal elements. Then an essentially normal element $x \in M(A)$ has the form $x=y+a$, where $y \in M(A)$ is normal and $a \in A$ if and only if $\Gamma(x)=0$.

Corollary 4.9 (cf. [Ln6, 11.7]). If $A$ is a finite matroid algebra, then every essentially normal element $x \in M(A)$ can be written as $x=y+a$, where $y \in M(A)$ is normal and $a \in A$.

Proof. We notice the fact that if $A$ is a finite matroid algebra, $K_{1}(M(A) / A)=0$ (see [Ell1]).

\section{REFERENCES}

[Bl] B. Blackadar, $K$-Theory for Operator Algebra, Springer Verlag, New York and Berlin, 1986. MR 88g: 46082

[BBEK] B. Blackadar, O. Bratteli, G. A. Elliott and A. Kumjian, Reduction of real rank of inductive limit $C^{*}$-algebras, Math. Ann. 292 (1992) 121-161. MR 93a:461112

[BDR] B. Blackadar, M. Dadarlat and M. R $\phi$ rdam, The real rank of inductive limit $C^{*}$-algebras, Math. Scand. 69 (1992) 211-216. MR 93e:46067

[BH] B. Blackadar and D. Handelman, Dimension functions and traces on $C^{*}$-algebras, J. Funct. Anal. 45 (1982), 297-340. MR 83g:46050

[BKR] B. Blackadar, A. Kumjian and M. R $\phi$ rdam, Approximately central matrix units and structure of noncommutative tori, K-theory 6 (1992). MR 93i:46129

[Bn1] L. G. Brown, Stable isomorphism of hereditary subalgebras, Pacific J. Math. 71 (1977), 335-348. MR 56:12894

[Bn2] L. G. Brown, Interpolation by projections in $C^{*}$-algebras of real rank zero, J. Operator Theory, 26 (1991), 383-387. MR 94j:46054

[BDF1] L. Brown, R. Douglas and P. Fillmore, Unitary equivalence modulo the compact operators and extensions of $C^{*}$-algebras, Proc. Conf. on Operator Theory, Springer Lecture Notes in Math. 345(1973),58-128. MR 52:1378

[BDF2] L. Brown, R. Douglas and P. Fillmore, Extensions of $C^{*}$-algebras and $K$-homology, Ann. of Math. 105(1977), 265-324. MR 56:16399

[BP] L. G. Brown and G. K. Pedersen, $C^{*}$-algebras of real rank zero, J. Funct. Anal., 99 (1991), 131-149. MR 92m:46086

[Dix] J. Dixmier, On some $C^{*}$-algebras considered by Glimm, J. Funct. Anal. 1 (1967) 183-203. MR 35: 4740

[Eff] E. Effros, Dimensions and $C^{*}$-algebras, CBMS Regional Conf. Ser. in Math., no. 46, Amer. Math. Soc., Providence, R. I., 1981, pp. 1-74. MR 84k:46042

[El11] G. A. Elliott, Derivations of Matroid $C^{*}$-algebras, II, Ann. of Math. (2) 100 (1974) 407-422. MR 50:5485

[El12] G. A. Elliott, The classification of $C^{*}$-algebras of real rank zero, J. Reine Angew. Math. 443 (1993), 179-219. MR 94i:46074

[El13] G. A. Elliott, A classification of certain simple $C^{*}$-algebras, pp. 373-385 in: Quantum and Non-Commutative Analysis (edited by H. Araki et al.), Kluwer, Dordrecht, 1993. MR 95h: 46089

[EE] G. A. Elliott and D. E. Evans, The structure of the irrational rotation $C^{*}$-algebras, Ann. Math. 138 (1993), 477-501. MR 94j:46066

[EGLP] G. A. Elliott, G. Gong, H. Lin and C. Pasnicu, Abelian $C^{*}$-algebras of $C^{*}$-algebras of real rank zero and inductive limits, Duke J. Math., to appear.

[EH] G. A. Elliott and D. E. Handelman, Addition of $C^{*}$-algebra extensions, Pacific J. Math., 137(1989) 87-121. MR 90e:46047

[EL] G. A. Elliott and T. Loring, AF embeddings of $C\left(T^{2}\right)$ with prescribed $K$-theory, J. Funct. Anal., 103 (1992), 1-25. MR 93b:46134

[G] K. R. Goodearl, Notes on a class of simple $C^{*}$-algebras with real rank zero, Publ. Mat. (Barcelona) 36 (1992), 637-654. MR 94f:46092

[Ln1] H. Lin, Ideals of multiplier algebras of simple $A F-C^{*}$-algebras, Proc. Amer. Math. Soc., 104 (1988), 239-244. MR 89j:46065

[Ln2] H. Lin, Simple $C^{*}$-algebras with continuous scales and simple corona algebras, Proc. Amer. Math. Soc., 112 (1991), 871-880. MR 92e:46118 
[Ln3] H. Lin, Generalized Weyl-von Neumann theorems, International J. Math. 2 (1991), 725739. MR 92m: 46087

[Ln4] H. Lin, Generalized Weyl-von Neumann theorems II, Math. Scand., 77 (1995), 599-616. CMP 96:05

[Ln5] H. Lin, Extensions by $C^{*}$-algebras with real rank zero, International J. Math., 4 (1993) 231-252. MR 94h:46095

[Ln6] H. Lin, $C^{*}$-algebra Extensions of $C(X)$, Mem. Amer. Math. Soc., 115 (1995), no. 550. MR 96b:46098

[Ln7] H. Lin, Exponential rank of $C^{*}$-algebras with real rank zero and Brown-Pedersen's conjecture, J. Funct. Anal., 114 (1993), 1-11. MR 95a:46079

[Ln9] H. Lin, Approximation by normal elements with finite spectra in simple AF-algebras, J. Operator Theory, 31 (1994), 83-98. MR 96b:46077

[Ln10] H. Lin, Notes on $K$-theory for multiplier algebras and corona algebras, preprint.

[Ln11] H. Lin, Approximation by normal elements with finite spectra in $C^{*}$-algebras of real rank zero, Pacific J. Math., 173 (1996), 443-489. CMP 96:14

[Ln12] H. Lin, Extensions by $C^{*}$-algebras of real rank zero II, Proc. London Math. Soc., 71 (1995), 641-674. CMP 95:17

[Ln13] H. Lin, Extensions by $C^{*}$-algebras with real rank zero III, preprint.

[Ln14] H. Lin, Equivalent open projections and their corresponding hereditary $C^{*}$-subalgebras, J. London Math. 41 (1990), 295-301. MR 91h:46101

[LR] H. Lin and M. Rørdam, Extensions of inductive limits of circle algebras, J. London Math., 51 (1995), 603-613. CMP 95:13

[LS] T. Loring and J. Spielberg, Approximation of normal elements in the multiplier algebra of an AF-algebra, Proc. Amer. Math. Soc. 121 (1994), 1173-1174. MR 94k:46116

[L] T. Loring, Normal elements of $C^{*}$-algebras of real rank zero without finite-spectrum approximants, J. London Math. Soc. 51 (1995), 353-364. MR 96b:46099

[M] G. J. Murphy, Diagonality in $C^{*}$-algebras, Math. Z., 199 (1988), 279-284. MR 90c:46070

[MS] J. A. Mingo and J. S. Spielberg, The index of normal Fredholm elements of $C^{*}$-algebras, Proc. Amer. Math. Soc. 113 (1991), 187-192. MR 91k:46081

[R] M. R $\phi$ rdam, Classification of inductive limits of Cuntz algebras, J. reine angew. Math. 440 (1993), 175-200. MR 94k:46120

[Pd1] G. K. Pedersen, $C^{*}$-Algebras and their Automorphism Groups, Academic Press, London/New York, 1979. MR 81e:46037

$[\mathrm{Pd} 2] \quad$ G. K. Pedersen, $S A W^{*}$-algebras and corona $C^{*}$-algebras, contributions to noncommutative topology, J. Operator Theory, 15 (1986),15-32. MR 87a:46095

[Pd3] G. K. Pedersen, The linear span of projections in simple $C^{*}$-algebras, J. Operator Theory, 4 (1980), 289-296. MR 82b:46078

[Ph] N. C. Phillips, Simple $C^{*}$-algebras with property weak (FU), Math. Scand., 69 (1991), 127-151. MR 93d:46121

[Zh1] S, Zhang, $K_{1}$-groups, quasidiagonality and interpolation by multiplier projections, Trans. Amer. Math. Soc. 325 (1991), 793-818. MR 91j:46069

[Zh2] S. Zhang, $C^{*}$-algebras with real rank zero and their corona and multiplier algebras, Part III, Canad. J. Math. 62 (1990), 159-190. MR 94i:46095

[Zh3] S. Zhang, $C^{*}$-algebras with real rank zero and their multiplier algebras, Part I, Pac. J. Math., 155 (1992), 169-197. MR 94i:46093

[Zh4] S. Zhang, A Riesz decomposition property and ideal structure of multiplier algebras, J. Operator Theory, 24(1990), 209-225. MR 93b:46116

[Zh5] S. Zhang, Diagonalizing projections in multiplier algebras over $C^{*}$-algebra, Pacific J. Math. 145(1990),181-200. MR 92h:46088

[Zh6] S. Zhang, On the structure of projections and ideals of corona algebras, Can. J. Math. 41(1989),721-742. MR 90h:46094

[Zh7] S. Zhang, Trivial $K_{1}$-flow of AF-algebras and finite von Neumann algebras, J. Funct. Anal., 92 (1990), 77-91. MR 92f:46086

Department of Mathematics, East China Normal University, Shanghai 20062, China Current address: Department of Mathematics, University of Oregon, Eugene, Oregon 974031222

E-mail address: lin@bright.uoregon.edu 